\title{
SKI Oncoprotein
}

National Cancer Institute

\section{Source}

National Cancer Institute. SKI Oncoprotein. NCI Thesaurus. Code C19993.

The c-SKI proto-oncogene encodes a transcriptional repressor that homo- and heterodimerizes with the related protein, snoN. SMAD3 associates with nuclear SKI protein in response to T GFB1 signaling, repressing transcriptional activation by SMAD3, and rendering cells resistant to the growth-inhibitory effects of TGFB. The effects of SKI can be overcome by increased expression of SMAD3. (from OMIM 164780 and NCI) 